\title{
PERCEPÇÃO DOS CUSTOS PERDIDOS: ESTUDO COM ALUNOS DO CURSO DE CIÊNCIAS CONTÁBEIS
}

\author{
PERCEPTION OF SUNK COSTS: APPLIED STUDY WITH ACCOUNTING STUDENTS
}

\author{
Luiz Medeiros de Araujo Neto \\ medeiros2002@hotmail.com \\ UNB \\ Isabel Cristina Henriques Sales \\ isabel.sales@gmail.com \\ UNB
}

\author{
Fátima de Souza Freire \\ ffreire51@gmail.com \\ UNB \\ Quintiliano da Silva Neiva Júnio \\ kintiliano@gmail.com \\ UNBC
}

\section{RESUMO}

No processo de tomada de decisão, pela ótica racional, os custos perdidos devem ser desconsiderados, pois incorridos no passado, tais custos não deveriam exercer qualquer efeito em uma decisão futura. Dado as diversas situações existentes nas empresas, empiricamente ou de forma subjetiva os administradores incluem em suas análises os custos perdidos. No entanto, há a possibilidade também de gestores com formação específica em finanças ou áreas correlatas não decidirem de maneira racional sobre os custos gerados nas empresas, estando propensos a tais falhas cognitivas. A pesquisa tem como objetivo verificar se alunos do curso de Ciências Contábeis perceberiam a existência dos custos perdidos em suas atividades corriqueiras. Observa-se também se noções de custos, experiência intelectual e gênero podem influência em tal percepção. $O$ estudo justifica-se face à necessidade de se conhecer a qualidade da tomada de decisão por pessoas com conhecimento em finanças e contabilidade. Foram aplicados 116 questionários a estudantes de Ciências Contábeis da Universidade de Brasília no período de outubro e novembro de 2012. Conclui-se que os estudantes são sensíveis aos custos perdidos, além disso, experiência profissional e gênero podem influenciar no processo de decisão. Já o fato de ter cursado a disciplina de Controladoria não apresenta influência na decisão de suas escolhas.

Palavras-chave: Custos Perdidos. Contabilidade Comportamental. Contabilidade Gerencial. Educação Contábil.

\begin{abstract}
In the process of making decision by the rational perspective, the sunk costs should be disregarded because it incurred in the past, and such costs shouldn't have any effect on a future decision. In the different situations in the companies, empirically or subjectively managers include the sunk costs in their analysis. However, there is also the possibility of managers with specific training in finance or related areas not decide rationally about the generated costs in the companies, being prone to such cognitive failures. The paper aims to determine whether students of Accounting realize the existence of sunk costs in their ordinary activities. There are also notions of costs, intellectual experience and gender may influence this perception. The survey was submitted to accounting students in the University of Brasília between October and November 2012, to examine the quality of decision making by people with expertise in finance and accounting. We conclude that students are sensitive to sunk costs, moreover, experience and gender can influence the decision process. However, the fact
\end{abstract}


that students have knowledge in cost management seems to have no influence on the decision of their choices.

Keywords: Sunk Costs. Behavioral Accounting. Managerial Accounting. Accounting Education.

Artigo recebido em: 10.04.2014; Aceito em: 07.07.2014

\section{INTRODUÇÃO}

A teoria econômica oferta de maneira quase inequívoca a análise marginal dos custos como principal fonte para as decisões de maximização do lucro e, logo, também como suporte para fundamentação dos preços. Os custos já incorridos, também conhecidos como custos perdidos, ou ainda sunk costs, embora possuam resultado marginal tendendo a zero, são importantes para as decisões de entrada e saída em investimentos pelos tomadores de decisão dentro das corporações.

As empresas, diante da grande competitividade e dinamismo presente na economia contemporânea, demandam a cada dia melhores processos de decisão em sua gestão. Segundo Souza, Avelar e Boina (2008), o dinamismo unido à volatilidade dos mercados, os quais são crescentes, podem ameaçar a sobrevivência das empresas.

Para que haja mais segurança nas decisões empresariais é preciso que os profissionais de controladoria estejam continuamente qualificados e capacitados, de forma a gerar informações relevantes para a tomada de decisão. Horngren (1985) enumera como funções do controller: planejamento para o controle, relatórios e interpretação, avaliação e assessoramento, gestão tributária, relatórios para o governo, proteção de ativos e avaliação econômica.

Em frente ao processo de decisão, o administrador da firma utiliza os custos relevantes ao processo, os quais são compostos pelos custos futuros de cada opção. Em geral esses custos futuros são comparados com as receitas futuras geradas, sendo escolhida a opção com maior expectativa de caixa futuro. Assim, os custos perdidos não devem ser considerados nos processos decisórios (SOUZA; SILVA; DOMINGOS, 2008). Na medida em que o custo é perdido, não deve ele exercer qualquer influência em decisões futuras e também não deve ser considerado dentro de uma escolha racional (MARTINS, 2001).

Com exemplo, considere uma situação de um aporte de 1 milhão de reais em um projeto de investimento, sendo demandado mais um aporte de 300 mil reais para sua conclusão. Um novo processo permite que o projeto seja executado por 200 mil reais com a mesma qualidade e que seja concluído no mesmo tempo. A decisão mais racional seria a de abandonar o projeto inicial e aderir à nova tecnologia.

No entanto, os agentes tomadores de decisões não são necessariamente racionais, podendo ser conduzidos a decisões equivocadas. Para Souza, Silva e Domingos (2008) a resistência de o agente de desprender-se de investimentos já incorridos é real, dado a escassez de recursos. Isso é nomeado por Arkes e Ayton (1999) como "don't waste rule” (regra do não desperdício, tradução nossa). Esses pesquisadores sugerem, inclusive, que a generalização da situação é visualizada a partir do efeito do custo perdido.

Com base no contexto apresentado, o presente artigo tem por objetivo testar a possível influência dos custos perdidos em estudantes de Ciências Contábeis e, além disso, sugerir alguns fatores que possam contribuir para minimizá-los. A pesquisa se justifica tendo em vista o fato de que os estudantes de Ciências Contábeis da atualidade serão os futuros tomadores de decisão, assim devem estar sendo treinados para tanto. 
O presente artigo está estruturado em cinco seções, incluindo esta introdução. Na segunda seção é apresentada uma breve revisão de literatura, a qual engloba a Teoria dos Prospectos, o efeito framing e os custos perdidos, todos aplicados ao ambiente gerencial. Na terceira seção, têm-se os procederes metodológicos aplicados à pesquisa, tais como o método de aplicação dos questionários e a justificativa para cada técnica aplicada na pesquisa. Quanto à quarta seção, apresenta-se a análise dos resultados obtidos, na qual se constata influência dos custos perdidos sob os alunos. Finalmente, na quinta e última seção, expõem-se os pontos conclusivos e sugestões para pesquisas futuras.

\section{REFERENCIAL TEÓRICO}

Os paradigmas teóricos comportamentais defendidos pela economia clássica se mostram conflitantes com o que se observa no mundo prático. Para a economia clássica os agentes, em seu processo de decisão, buscam exclusivamente a maximização de sua utilidade. Em oposição a isso, Kahneman e Tversky (1979) sugerem uma nova forma de visualizar o processo decisório sob a incerteza, a qual é nomeada como a Teoria dos Prospectos. A partir de experimentos, a Teoria dos Prospectos aponta violações constantes dos axiomas microeconômicos de comportamento racional (CARVALHO JUNIOR; ROCHA; BRUNI, 2009). Diversas pesquisas que antecederam a Teoria dos Prospectos frisaram a possibilidade de reversões de preferências entre as alternativas de escolhas ao modificar-se a apresentação do problema. Tversky e Kahneman (1981) nomearam esse fenômeno como Efeito Framing, o qual é explicado pela Teoria dos Prospectos. Os autores explanam que os efeito dos Custos Perdidos são observáveis, no mercado de capitais, em decisões individuais de investimento.

Heath (1995) analisou o efeito dos Custos Perdidos numa ótica temporal - os resultados mostram que os Custos Perdidos exerceram efeito nas escolhas orçamentárias dos investidores. Quanto aos problemas que ocorrem em decisões estratégicas e operacionais, baseadas em informações fornecidas pela Controladoria das empresas, observa-se a presença do Efeito Framing (RUTLEDGE, 1995). Para Carvalho Junior Rocha, Bruni, (2009) o Efeito Framing pode ocorrer em situações diversas, inclusive em decisões internas.

$\mathrm{Na}$ contabilidade, focar as finanças comportamentais é importante para que se entenda a motivação dos agentes envolvidos nas tomadas de decisões. Ligar a contabilidade a teorias psicológicas e de biologia neurológica ajudam a interpretar de forma prática as teorias financeiras.

Koppel (2011) explana que a "elevação do comprometimento" (escalation of commitment) é como uma armadilha mental que pode influenciar decisões de investimento. $\mathrm{O}$ fenômeno se refere a uma situação na qual as pessoas tomam decisões irracionais com base em decisões racionais passadas. Outro termo utilizado para esse comportamento, conforme Koppel (2011) é "falácia dos custos perdidos" (sunk cost fallacy).

McMahon (2010) apresenta os custos perdidos de uma forma mais objetiva, ao afirmar que essa falácia ocorre quando os individues permitem que suas escolhas entre alternativas futuras sejam afetadas pelos custos que ocorreram em algum momento passado - custos estes que não podem ser alterados, ou "recuperados". A Moderna Teoria de Finanças defende que apenas benefícios e custos incrementais devem afetar a tomada de decisão. De tal modo, considerar os custos perdidos é uma atitude irracional. Garrison, Noreen e Brewer (2007) destacam, também, o sinônimo "custo irrecuperável" - um custo que já ocorreu e não pode mais ser alterado, o que os torna custos não diferenciais.

No Brasil, as pesquisas com foco em finanças comportamentais são recentes e ainda escassas. Sobre custos perdidos podemos, todavia, citar: Murcia e Borba (2006) que verificaram se estudantes eram influenciados pela falácia ao responderem um questionário com cinco 
cenários de decisão. Com base na amostra percebeu-se que os alunos não estavam criticamente preparados para o desafio e cederam à irracionalidade do experimento.

Ainda, Silva e Domingos (2010) objetivaram determinar as variáveis que influenciavam a insistência irracional; Souza, Silva e Domingos (2008) estudaram se a informação do valor investido num determinado projeto influenciava, ou não, o efeito do custo perdido. Araujo Neto e Freire (2012) encontraram quebra dos axiomas econômicos clássicos em estudantes de graduação de contabilidade quando sujeitos a decisões de investimentos.

\section{PROCEDER METODOLÓGICO}

O trabalho tem como propósito reaplicar na Universidade de Brasília (UnB) os estudos de Murcia e Borba (2006) realizados no Brasil, mais precisamente no Estado de Santa Catarina, além das pesquisas de Arkes e Blumer (1985) e Harrison e Shanteau (1993) realizadas no exterior.

Quanto ao levantamento dos dados foi utilizado um questionário, o qual é baseado no trabalho de Murcia e Borba (2006). Mattar (1999) acredita que essa técnica é a mais eficaz em pesquisas para a medição de erros cognitivos. Primeiramente, foram realizadas alterações no questionário original [o aplicado por Múrcia e Borba (2006)], de forma a adaptá-lo a realidade dos alunos da UnB. De tal modo, foi elaborado um questionário com cinco questões posteriormente apresentado na análise de resultados.

Quanto às características da amostra, foram aplicados um total de 116 questionários, sendo que exatamente a metade dos participantes havia cursado a disciplina Controladoria. $\mathrm{Na}$ Universidade de Brasília, essa é a disciplina que abarca os conteúdos relativos a Sunk Costs. Com relação ao gênero, não houve predominância entre os respondentes, sendo 59\% do sexo masculino. Em média foram selecionados respondentes com boa maturidade financeira, sendo a amostra composta por estudantes do quarto ao décimo segundo semestre (há de se salientar que alunos cursando o décimo segundo semestre estão atrasados em relação ao fluxo médio esperado e sugerido).

Em relação a idade foi notado que, em média, os estudantes possuem 23,4 anos, dentro de um intervalo 18 a 44 anos. Da amostra, 92\% não estagiou ou estagia na área de Contabilidade Gerencial, mostrando que apenas uma minoria, $8 \%$, trabalha ou estagia na área.

Como mencionado anteriormente, a pesquisa procura testar a sensibilidade dos alunos de graduação em Contabilidade da Universidade de Brasília aos Custos Perdidos e, ainda, determinar, dentre três fatores, quais teriam maior impacto na percepção desses custos. Esses três fatores a serem testados são: gênero, conhecimento teórico em Contabilidade Gerencial e pratica em Contabilidade Gerencial. Para se testar tais constructos foram utilizadas proxies, as quais são definidas no quadro 1 , a seguir.

Quadro 1: Proxies utilizadas na pesquisa

\begin{tabular}{|c|c|}
\hline Constructo & Proxy \\
\hline Gênero & Sexo (masculino e feminino) \\
\hline Conhecimento Teórico & Ter Cursado a Disciplina Controladoria \\
\hline Pratica em Contabilidade Gerencial & Trabalhar ou Estagiar na Área \\
\hline
\end{tabular}

Fonte: Elaborado pelos autores (2012)

A escolha se justifica a partir de pesquisas anteriores que encontraram evidencias fortes para o uso de tais proxies. São elas: Fish (2012) para diferença entre os sexos, Carvalho Júnior Rocha; Bruni, (2009) para experiência profissional e conhecimento intelectual. 
Os dados foram tabulados no software excel e todas as estatísticas foram geradas por meio do programa SPSS (Statistical Package for Social Sciences). Para testar a sensibilidade aos custos perdidos, assim como qual dos elementos teria maior influencia na percepção de tais custos, foram aplicados testes de proporções, mais especificamente o teste Qui-quadrado $\left(\chi^{2}\right)$. Hoffmann (2009) sugere que o teste Qui-quadrado é bastante adequado para aplicar testes de hipóteses sobre proporções. Por ser um teste não-paramétrico, o Qui-quadrado não pressupõe normalidade nas variáveis testadas, assim não foi feito nenhum teste de ajustamento nas variáveis em estudo. Para os testes executados, o nível de significância arbitrado foi de 0,05 por se tratar do valor usual em pesquisas científicas em ciências sociais aplicadas (GUJARATI, 2006).

\section{RESULTADOS E ANÁLISES}

Apresenta-se a seguir cada uma das cinco perguntas que constavam no questionário, seguidas de suas devidas analises. A partir dos dados obtidos, realizou-se uma breve análise descritiva, nas quais foram arredondados todos os percentuais para fins didáticos. Em seguida o teste Qui-quadrado foi aplicado nas variáveis em estudo.

\subsection{Promoção Supermercado}

A primeira pergunta está descrita a seguir:

No regresso do trabalho, você decide parar no supermercado perto de sua casa e comprar uma lasanha pequena (apenas para uma pessoa) que está com o preço promocional de $\mathrm{R} \$ 12$. Antes de colocar a lasanha no forno, você decide ligar para um amigo e convidá-lo para ir para a sua casa para assistir a final do campeonato de basquete. Seu amigo confirma a ida, então você volta ao mesmo supermercado para comprar uma segunda pequena lasanha. Ao chegar ao supermercado você percebe que as lasanhas pequenas - em promoção - se esgotaram. $\mathrm{O}$ produto agora custa $R \$ 25$ reais.

Você realiza a sua compra e, já em casa, coloca as duas lasanhas no forno. Minutos depois, você recebe uma ligação de seu amigo dizendo que um imprevisto o impossibilitará de comparecer ao encontro.

Você sabe não está com fome suficiente para comer as duas lasanhas. Não há a possibilidade de deixar as sobras para o dia seguinte ou mesmo recongelar. A solução é jogar uma das lasanhas no lixo.

As duas lasanhas são idênticas (do mesmo tamanho e sabor). Qual a sua decisão?

A. Comer a lasanha que custou $R \$ 12$ reais.

B. Comer a lasanha que custou $\mathrm{R} \$ 25$ reais.

C. Tanto faz.

Tabela 1: Estatísticas Descritivas para Questão 1

\begin{tabular}{cccccccc}
\hline & \multirow{2}{*}{ Geral } & \multicolumn{2}{c}{ Estagia/ trabalha } & \multicolumn{2}{c}{ Cursou Controladoria } & \multicolumn{2}{c}{ Gênero } \\
& & Sim & Não & Sim & Não & Masculino & Feminino \\
\hline Lasanha R\$ 12 & $4(3 \%)$ & $0(0 \%)$ & $4(4 \%)$ & $1(2 \%)$ & $3(5 \%)$ & $3(4 \%)$ & $1(2 \%)$ \\
Lasanha R\$ 25 & $24(21 \%)$ & $1(11 \%)$ & $23(21 \%)$ & $9(16 \%)$ & $15(26 \%)$ & $13(19 \%)$ & $11(23 \%)$ \\
Tanto faz & $88(76 \%)$ & $8(89 \%)$ & $80(75 \%)$ & $48(83 \%)$ & $40(69 \%)$ & $52(76 \%)$ & $36(75 \%)$ \\
\hline \multirow{2}{*}{ Total } & $\mathbf{1 1 6}$ & $\mathbf{9}$ & $\mathbf{1 0 7}$ & $\mathbf{5 8}$ & $\mathbf{5 8}$ & $\mathbf{6 8}$ & $\mathbf{4 8}$ \\
& $(\mathbf{1 0 0 \% )}$ & $\mathbf{( 1 0 0 \% )}$ & $\mathbf{( 1 0 0 \% )}$ & $\mathbf{( 1 0 0 \% )}$ & $\mathbf{( 1 0 0 \% )}$ & $(\mathbf{1 0 0 \% )}$ & $(\mathbf{1 0 0} \%)$ \\
\hline
\end{tabular}


Fonte: Elaborado pelos autores (2012)

Para a questão 1 espera-se como resposta a alternativa "tanto faz", pois como todos os custos foram incorridos, já não faz diferença qual lasanha será comida. Como é possível interpretar a partir da tabela 1, 24\% dos alunos que participaram da pesquisa mostram sensibilidade aos custos perdidos no ambiente apresentado. Dentre os 24\%, 3\% comeriam a lasanha de 12 reais e $21 \%$ comeriam a lasanha de 25 reais.

A análise descritiva sugere que tanto a experiência profissional quanto a intelectual interferiram na percepção dos alunos quanto aos custos perdidos. Foi observado que $89 \%$ dos que possuem experiência profissional na área gerencial não apresentaram sensibilidade, contra $75 \%$ dos que não possuem experiência.

Quanto ao fato de cursar a disciplina Controladoria, $83 \%$ dos matriculados na matéria se mostraram livres da influencia dos sunk costs, contra $69 \%$ dos que não estudaram o assunto. O gênero não apresentou diferença significativa - $24 \%$ dos homens se demonstraram sensíveis, enquanto $25 \%$ das mulheres apresentaram o mesmo comportamento.

No entanto, a análise não paramétrica, com p-value de 0,001 , sugere que apenas a experiência profissional na área gerencial influenciou a sensibilidade aos custos perdidos. A experiência intelectual e o gênero, com $p$-values de 0,40 e 0,09 respectivamente, não foram considerados influentes na questão 1 - não são significantes a $5 \%$.

\subsection{Carona Para Casa}

A segunda pergunta está descrita a seguir:

Você é estudante da UnB, mora em Brasília e pretende visitar seus pais em Goiânia (GO) nas férias de julho. Como você não possui um automóvel, tenta então encontrar na universidade alguém que possa te oferecer carona. Depois de muito procurar, você desiste e decide ir de ônibus. Então, você compra a passagem por R \$ 60. Momentos antes da viagem, você encontra um conhecido que te oferece uma carona gratuita no mesmo horário em seu ônibus sairia para Goiânia. Você não pode mais vender nem tampouco devolver a passagem de ônibus para a empresa. Qual a sua decisão?

A. Ir de ônibus.

B. Ir de carona com o conhecido.

Tabela 2: Estatísticas Descritivas para Questão 2

\begin{tabular}{cccccccc}
\hline & \multirow{2}{*}{ Geral } & \multicolumn{2}{c}{ Estagia/ trabalha } & \multicolumn{2}{c}{ Cursou Controladoria } & \multicolumn{2}{c}{ Gênero } \\
& & Sim & Não & Sim & Não & Masculino & Feminino \\
\hline Ônibus & $38(33 \%)$ & $5(56 \%)$ & $33(31 \%)$ & $20(34 \%)$ & $18(31 \%)$ & $19(28 \%)$ & $19(40 \%)$ \\
Carona & $78(67 \%)$ & $4(44 \%)$ & $74(69 \%)$ & $38(66 \%)$ & $40(69 \%)$ & $49(72 \%)$ & $29(60 \%)$ \\
\hline Total & $\mathbf{1 1 6 ( 1 0 0 \% )}$ & $\mathbf{9} \mathbf{( 1 0 0 \% )}$ & $\mathbf{1 0 7}(\mathbf{1 0 0 \%})$ & $\mathbf{5 8 ( 1 0 0 \% )}$ & $\mathbf{5 8} \mathbf{( 1 0 0 \% )}$ & $\mathbf{6 8} \mathbf{( 1 0 0 \% )}$ & $\mathbf{4 8} \mathbf{( 1 0 0 \% )}$ \\
\hline \multicolumn{3}{c}{ Fonte: Elaborado pelos autores (2012) }
\end{tabular}

Na questão 2 espera-se como resposta a alternativa "ir de carona", pois considerando-se todos os custos incorridos, ir de carona com um amigo apresenta-se mais agradável, além de ter sido a primeira opção.

A tabela 2, acima, ilustra que $33 \%$ dos alunos se mostraram afetados pelos sunk costs. A partir das estatísticas descritiva percebe-se que para a questão 2 tanto a experiência profissional quanto o gênero interferiram na percepção dos alunos. Como resultado, é observado que 44\% 
dos que possuem experiência profissional na área gerencial não apresentaram sensibilidade, contra 69\% dos que não possuem experiência, indo contra o resultado da questão 1.

Quanto à disciplina Controladoria, 66\% dos que cursaram se mostram livres da influencia dos sunk costs contra $69 \%$ dos que não estudaram o assunto. O gênero apresenta agora uma diferença mais visível, com $28 \%$ dos homens sensíveis, contra $40 \%$ das mulheres com a mesma atitude.

Com p-values de 0,692, 0,129 e 0,188 o teste qui-quadrado evidencia, para a situação simulada acima, que o fato de o aluno ter cursado a disciplina Controladoria, ter experiência profissional na área gerencial e o gênero não apresentaram influencia em sua escolha para a resposta. Esses valores sugerem que não deve haver, com um bom nível de confiança, influência das variáveis testadas.

\subsection{Viagem Para os Estados Unidos}

A seguir, as informações relativas à questão 3 do questionário:

Você está escutando um programa de música da rádio Jovem Pan quando o locutor do programa liga para a sua casa, afirmando que você acaba de ganhar uma viagem, com todas as despesas pagas, para passar uma semana em um hotel nos Estados Unidos. Você pode escolher entre dois destinos: São Francisco (SF) ou Nova Iorque (NY). Você prefere ir para São Francisco mas, antes de decidir, liga para um agente de viagens e descobre que o preço de um pacote de fim de semana para Nova Iorque custa $R \$ 2.500$. Para a cidade de São Francisco, o valor cai para $\mathrm{R} \$ 1.750$. Você precisa decidir por um dos dois destinos. Qual a sua decisão?

A. Ir para Nova Iorque

B. Ir para São Francisco

Tabela 3: Estatísticas Descritivas para Questão 3

\begin{tabular}{cccccccc}
\hline & \multirow{2}{*}{ Geral } & \multicolumn{2}{c}{ Estagia/ trabalha } & \multicolumn{2}{c}{ Cursou Controladoria } & \multicolumn{2}{c}{ Gênero } \\
& & Sim & Não & Sim & Não & Masculino & Feminino \\
\hline Ir para NY & $58(50 \%)$ & $4(44 \%)$ & $54(50 \%)$ & $30(52 \%)$ & $28(48 \%)$ & $33(49 \%)$ & $25(52 \%)$ \\
Ir para SF & $58(50 \%)$ & $5(56 \%)$ & $53(50 \%)$ & $28(48 \%)$ & $30(52 \%)$ & $35(51 \%)$ & $23(48 \%)$ \\
\hline \multirow{2}{*}{ Total } & $\mathbf{1 1 6}$ & $\mathbf{9}(\mathbf{1 0 0 \% )}$ & $\mathbf{1 0 7}$ & $\mathbf{5 8 ( 1 0 0 \% )}$ & $\mathbf{5 8}(\mathbf{1 0 0 \% )}$ & $\mathbf{6 8}$ & $\mathbf{4 8}(\mathbf{1 0 0 \%})$ \\
& $(\mathbf{1 0 0 \% )}$ & & $\mathbf{( 1 0 0 \% )}$ & & & $(\mathbf{1 0 0 \% )}$ & \\
\hline
\end{tabular}

Fonte: Elaborado pelos autores (2012)

Dado que o respondente prefere ir para São Francisco, a primeira alternativa seria a mais adequada, pois todos os custos foram incorridos. No entanto, conforme exposto na tabela 3 , metade dos alunos optou por Nova Iorque, sendo então afetados pelos custos perdidos. A análise descritiva sustenta haver diferenças mínimas entre as respostas, em todas as variáveis estudadas. O teste Qui-quadrado sugere que, tanto o gênero, quanto a disciplina Controladoria, com p-values de 0,07 e 0,76 respectivamente, não exerceram qualquer efeito nas escolhas dos estudantes. Já o trabalho ou estágio na área gerencial, com $p$-value 0,001 , mostrou afetar a decisão dos alunos.

\subsection{Show da Banda Preferida}

A seguir, as informações relativas à questão 4 do questionário: 
Você tem muito interesse em assistir um show de uma de suas bandas preferidas, que será realizado na cidade de Brasília. Entretanto, você não tem dinheiro suficiente para comprar o ingresso que está sendo vendido por $\mathrm{R} \$ 180$. Assim, você desiste de assistir ao show e compra um convite por $\mathrm{R} \$ 100$ reais para ir a uma boate na mesma noite. No dia programado, você recebe uma ligação de seu primo dizendo que a namorada dele terá que estudar e não irá mais acompanhá-lo ao show. Ele tem, portanto, um ingresso disponível, e te oferece gratuitamente. Contudo, já não há tempo para você vender ou devolver o convite da boate. Qual a sua decisão?
A. Ir ao Show.
B. Ir à Boate.

Tabela 4: Estatísticas Descritivas para Questão 4

\begin{tabular}{cccccccc}
\hline & \multirow{2}{*}{ Geral } & \multicolumn{2}{c}{ Estagia/ trabalha } & \multicolumn{2}{c}{ Cursou Controladoria } & \multicolumn{2}{c}{ Gênero } \\
& & Sim & Não & Sim & Não & Masculino & Feminino \\
\hline Show & $108(93 \%)$ & $8(89 \%)$ & $100(93 \%)$ & $56(97 \%)$ & $52(90 \%)$ & $65(96 \%)$ & $43(90 \%)$ \\
Boate & $8(7 \%)$ & $1(11 \%)$ & $7(7 \%)$ & $2(3 \%)$ & $6(10 \%)$ & $3(4 \%)$ & $5(10 \%)$ \\
\hline Total & $\mathbf{1 1 6 ( 1 0 0 \% )}$ & $\mathbf{9 ( 1 0 0 \% )}$ & $\mathbf{1 0 7}(\mathbf{1 0 0 \%})$ & $\mathbf{5 8 ( 1 0 0 \% )}$ & $\mathbf{5 8 ( 1 0 0 \% )}$ & $\mathbf{6 8 ( 1 0 0 \% )}$ & $\mathbf{4 8}(\mathbf{1 0 0 \%})$ \\
\hline \multicolumn{3}{c}{ Fonte: Elaborado pelos autores $(2012)$}
\end{tabular}

Com todos os custos incorridos é esperado que os alunos escolham a opção "ir para o show", pois era a intenção inicial como programação para aquela data. Na tabela 4 é evidenciado que 93\% dos alunos optaram por ir ao show ao invés da boate, mostrando que, para esta situação, foram poucos os afetados pelos sunk costs. A situação descrita nos remete que em um momento no qual há profundo conhecimento sobre o assunto, deve haver menor sensibilidade aos custos perdidos. Como é notado por meio das estatísticas descritiva, os percentuais mudam pouco entre as confrarias, demonstrando certo padrão de resposta para todos os grupos estratificados. O resultado do teste Qui-quadrado sugere que a experiência profissional e o gênero, com p-values de 0,001 e 0,03 respectivamente, interferiram na decisão, os respondentes que trabalham na área e as mulheres foram nesse caso mais sensíveis aos custos perdidos. A disciplina Controladoria, com p-value de 0,70, não apresentou significância estatística, sugerindo que para essa questão a disciplina não exerceu efeito sobre os respondentes.

\subsection{Máquina de Lavar}

Você mora sozinho em uma kitinet (pequeno apartamento) e gasta cerca de R \$ 120 mensais para limpar suas roupas em uma lavanderia local. Um dia você decide comprar uma máquina de lavar roupas, em promoção, por $\mathrm{R} \$ 1.000$. A máquina funciona perfeitamente, mas após dois meses de uso, você percebe que a conta de luz e de água aumentou aproximadamente $\mathrm{R} \$$ 120 reais por mês. Você não pode devolver a máquina e provavelmente não conseguirá revende-la já que existem outras máquinas operando com economia de água e luz. O dono da loja propõe, então, comprar a máquina por $\mathrm{R} \$ 400$. Qual a sua decisão?

A. Vende a máquina e volta a lavar sua roupa na lavanderia local.

B. Não vende a máquina e continua lavando a roupa em casa.

Tabela 5: Estatísticas Descritivas para Questão 5

Geral Estagia/ trabalha Cursou Controladoria Gênero




\begin{tabular}{cccccccc} 
& & Sim & Não & Sim & Não & Masculino & Feminino \\
\hline Vende & $67(58 \%)$ & $3(33 \%)$ & $64(60 \%)$ & $35(60 \%)$ & $32(55 \%)$ & $42(62 \%)$ & $25(52 \%)$ \\
Não vende & $49(42 \%)$ & $6(67 \%)$ & $43(40 \%)$ & $23(40 \%)$ & $26(45 \%)$ & $26(38 \%)$ & $23(48 \%)$ \\
\hline Total & $\mathbf{1 1 6 ( 1 0 0 \% )}$ & $\mathbf{9}(\mathbf{1 0 0 \%})$ & $\mathbf{1 0 7 ( 1 0 0 \% )}$ & $\mathbf{5 8 ( 1 0 0 \% )}$ & $\mathbf{5 8}(\mathbf{1 0 0 \% )}$ & $\mathbf{6 8 ( 1 0 0 \% )}$ & $\mathbf{4 8 ( 1 0 0 \% )}$ \\
\hline \multicolumn{6}{c}{ Fonte: Elaborado pelos autores (2012) }
\end{tabular}

Como o uso da máquina é indiferente, espera-se, como resposta racional a opção "vender a máquina". Na tabela 5, nota-se que $42 \%$ dos alunos em geral foram afetados pelos sunk costs. A partir da análise descritiva dos dados é observado que apenas $33 \%$ dos que possuem experiência profissional na área gerencial não foram afetados pelos custos perdidos, contra $60 \%$ dos que não possuem experiência.

Quanto à disciplina Controladoria, 60\% dos que cursaram se mostram livres da influencia dos sunk costs contra $55 \%$ dos que não a estudaram. Já o gênero apresentou $62 \%$ dos homens não afetados, enquanto $52 \%$ das mulheres também não foram afetadas. Com $p$-value de 0,45 , o teste Qui-quadrado sugere que a disciplina Controladoria não melhorou a percepção dos alunos quanto aos custos perdidos. No entanto, experiência profissional na área gerencial e o gênero do respondente, com p-values 0,001 e 0,02 respectivamente, representam duas variáveis com influência na decisão para a questão presente. A partir do teste é possível sugerir com certo rigor que, para esse caso específico, as mulheres e os profissionais da área se mostraram mais afetados pelos sunk costs.

\section{CONSIDERAÇÕES FINAIS}

O trabalho teve como objetivo analisar se alunos do curso de Ciências Contábeis seriam sensíveis aos custos perdidos e, ainda, determinar se a experiência profissional, a experiência acadêmica e o gênero poderiam influenciar em tal sensibilidade. Assim este trabalho difere-se de outros artigos que verificaram apenas a sensibilidade aos custos perdidos em estudantes. Buscou-se uma amostra em processo de formação na área de conhecimento econômico e contábil.

O resultado sugere que em algum grau, para todas as perguntas, estudantes de Ciências Contábeis acabaram por adicionar os custos perdidos em suas decisões. Quanto às proxies testadas, observou-se, todavia, não existir uma relação determinada. No entanto estudantes com experiência profissional na área gerencial obtiveram melhores resultados em quatro das cinco situações. Já com relação ao gênero, foram observadas significâncias estatísticas em duas das cinco questões e, nas duas ocasiões, os homens se mostraram menos propensos à falácia dos custos perdidos. A disciplina Controladoria não apresentou influência na decisão tomada nas questões propostas.

Há de se ressaltar que é importante, no ensino da contabilidade (assim como de áreas afins) que os vieses comportamentais sejam lecionados de forma eficaz para que os alunos adentrem o mercado como profissionais preparados para tomar decisões gerenciais racionais, que não sejam afetadas por algum viés.

Para futuras pesquisas, sugere-se o enfoque nas diferentes formas de ensino dos custos perdidos. Isso pode ser trabalhado por meio de um levantamento do currículo das Instituições de Ensino Superior, assim como por meio de entrevistas com professores de matérias correlatas e gestores dos departamentos de contabilidade, administração e economia para que seja efetivamente mapeada, analisada e melhorada (ou divulgada, caso apresente um nível satisfatório de aprendizado pelos discentes) visando uma aplicação nacional da melhor prática didática. 


\section{REFERÊNCIAS}

ARAUJO NETO, L. M.; FREIRE, F. S. Comportamento Manada: estudo aplicado em estudantes do curso de ciências Contábeis. Revista de Informação Contábil, vol. 7, n. 1, 2013.

ARKES, H.; AYTON, P. The Sunk Cost an Concorde Effects: Are Humans Less Rational Than Lower Animals? Psychological Bullettin, v. 125, 5, p. 591- 600, 1999.

ARKES, R; BLUMER, C. The Psychology of Sunk Costs. Organizational Behavior and Human Decision Process, v. 35, p. 124-140, 1985.

CARVALHO JUNIOR, C. V. O; ROCHA, J. S; BRUNI, A. L. O Aprendizado Formal de Controladoria e a Minimizaçao do Efeito Framing: Um Estudo de Decisões Gerenciais Envolvendo Custos de Oportunidades. Revista de Educação e Pesquisa em Contabilidade, v. 3, n. 3, pg 18-38, 2009.

SOUZA, F. A.; SILVA, C. A. T.; DOMINGOS, N. T. Efeito do custo perdido: a influências do custo perdido na decisão de investimento Revista de Contabilidade e Organizações, v. 2, n. 2 , jan./abr. 2008

FISH, J. Behavioral Finance: A Study of Gender Affects on Investing Decisions.

Disponível em: <http://purl.umn.edu/132175>. Acesso em: 25 out. 2012.

GARRISON, R. H.; NOREEN, E. W.; BREWER, P. C. Contabilidade Gerencial. 11. ed. Rio de Janeiro: LTC, 2007.

GUJARATI, D. Econometria Básica. Rio de Janeiro: Elsevier, 2006.

HARRISON, P; SHANTEAU, J. Do Sunk Costs Effects Generalize to Cost Accounting Students? Advances in Management Accounting. v. 2, p. 171-186, 1993.

HEATH, Chip. Escalation and de-escalation of commitment in response to sunk costs: the role of budgeting in mental accounting. Organizational Behavior and Human Decision Processes, v. 62, n.1, p.38-54, 1995.

HOFFMANN, R. Estatística para Economistas. São Paulo: Cengage Learning, 2009.

HORNGREN, C. T. Introdução à Contabilidade Gerencial. Rio de Janeiro: Prentice-Hall, 1985.

KAHNEMAN, D; TVERSKY, A. Prospect theory: an analysis of decision under risk. Econometrica. p. 263-291, 1979.

KOPPEL, Robert. Investing and the irrational mind. McGrawHill: Estados Unidos, 2011.

MARTINS, E. Contabilidade de custos. São Paulo: Atlas, 2001. 
MATTAR, F. N. Pesquisa de Marketing: metodologia, planejamento, execução e análise. 5 ed. São Paulo: Atlas, 1999.

MCMAHON, R. G. P. Behavioral finance: a background briefing. 2010. Research Paper Series. Disponível em: 〈http://www.flinders.edu.au/sabs/business/research/papers/05-9.pdf $>$. Acesso em: 18 set. 2012.

MURCIA, F., BORBA, J. A. Um Estudo Empírico Sobre os Efeitos dos Sunk Costs no Processo Decisório dos Indivíduos: Evidências dos Estudantes de Graduação de uma Universidade Federal. UnB Contábil, v. 9, n. 2, p. 223-247, 2006.

RUTLEDGE, R. W. The ability to moderate regency effects through framing of management accounting information. Journal of Management Issues, v. 7, n. 1, p. 27-40, 1995.

Science, v. 211, p. 453-458, 1981.

SILVA, C. A. T.; DOMINGOS, N. T. Sunk cost e insistência irracional: o comportamento face às decisões de alocações de recursos. Advances in Scientific and Applied Accounting, v. 3, p. 41-64, 2010.

SOUZA, A. A.; AVELAR, E. A.; BOINA, T. M. Gestão de Custos e Formação de Preços em Empresas de Produção por Encomendas: Estudos de Caso. Revista de Informação Contábil, v. 2, n. 1, p. 56-81, 2008.

TVERSKY, A; KAHNEMAN, D. The Framing of Decisions and the Psychology of Choice. Science, v. 211, n. 4481, p. 453-458, 1981. 